\title{
A review of potential applications of MR-guided focused ultrasound for targeting brain tumor therapy
}

\author{
Layton Lamsam, BS, ${ }^{1}$ Eli Johnson, BS, ${ }^{1}$ lan D. Connolly, MS, ${ }^{1}$ Max Wintermark, MD, ${ }^{2}$ and \\ Melanie Hayden Gephart, MD, MAS'1
}

Departments of ${ }^{1}$ Neurosurgery and ${ }^{2}$ Radiology, Stanford University Medical Center, Stanford, California

\begin{abstract}
Magnetic resonance-guided focused ultrasound (MRgFUS) has been used extensively to ablate brain tissue in movement disorders, such as essential tremor. At a lower energy, MRgFUS can disrupt the blood-brain barrier (BBB) to allow passage of drugs. This focal disruption of the BBB can target systemic medications to specific portions of the brain, such as for brain tumors. Current methods to bypass the BBB are invasive, as the BBB is relatively impermeable to systemically delivered antineoplastic agents. Multiple healthy and brain tumor animal models have suggested that MRgFUS disrupts the BBB and focally increases the concentration of systemically delivered antitumor chemotherapy, immunotherapy, and gene therapy. In animal tumor models, combining MRgFUS with systemic drug delivery increases median survival times and delays tumor progression. Liposomes, modified microbubbles, and magnetic nanoparticles, combined with MRgFUS, more effectively deliver chemotherapy to brain tumors. MRgFUS has great potential to enhance brain tumor drug delivery, while limiting treatment toxicity to the healthy brain.
\end{abstract}

https://thejns.org/doi/abs/10.3171/2017.11.FOCUS17620

KEY WORDS targeted drug delivery; MR-guided; image-guided; focused ultrasound; blood-brain barrier; brain tumor

$\mathrm{T}$ HE blood-brain barrier (BBB) protects the brain from fluctuations in plasma concentrations and toxins capable of disturbing neural function. The BBB also restricts the delivery of drugs to the brain, as it is impermeable to most biological and small-molecule therapeutics. ${ }^{1}$ Limitations in drug transport across the BBB has made treating CNS tumors difficult. ${ }^{37,48,49,52}$ Magnetic resonance-guided focused ultrasound (MRgFUS) uses acoustic waves in the ultrasound spectrum to transiently raise the temperature or activate molecules at a specific location. This emerging technology is used primarily for thermal ablation of central brain locations, such as for the treatment of essential tremor and Parkinson's disease. ${ }^{5,21,41,50}$ MRgFUS is an image-guided, noninvasive treatment option that can be used to manipulate the BBB and achieve focal CNS tumor drug delivery of systemically administered treatments.

\section{Background}

\section{The Blood-Brain and Blood-Tumor Barriers}

The BBB is composed of endothelial cells, astrocyte foot processes, and pericytes. ${ }^{4}$ Tight junctions between these cells form a barrier, preventing transcellular and paracellular diffusion of most substances from entering the brain. Multiple transport systems, enzymes, and receptors involved in the regulation of the BBB have been identified..$^{42}$ Tight regulation of the BBB maintains cerebral function by preventing the passive crossing of cells and molecules that are capable of inducing inflammation and damage to the CNS. ${ }^{13}$ Consequently, methods of bypassing the BBB for therapeutic effect should be targeted and transient to prevent global brain injury.

The blood-tumor barrier (BTB), located between the malignancy and blood vessels, is formed after the tumor is larger than $0.2 \mathrm{~mm}^{3} .^{32}$ As the tumors grow, astrocyte foot processes are displaced from blood vessels by malignant cells, leading to perpetual fenestration of the BBB..$^{58} \mathrm{Al}$ though the level of disruption often remains subtherapeutic, BTB-targeted drug delivery holds promise for effective treatment of brain tumors through increased permeability.

\section{Conventional Approaches to Drug Delivery in the Brain}

Ostrom et al. predicted that there would be more than 79,000 new cases of brain metastases and primary brain tumors in 2017 in the United States. ${ }^{44}$ Peripherally administered chemotherapy must cross the BTB to achieve a

ABBREVIATIONS BBB = blood-brain barrier; BCNU = bis-chloroethylnitrosourea; BTB = blood-tumor barrier; cLeDOX = cationic liposome-encapsulated doxorubicin; cMB = cationic microbubble; DOX = doxorubicin; FUS = focused ultrasound; IL = interleukin; LeDOX = liposome-encapsulated DOX; LePTX = liposome-enhanced paclitaxel; MNP = magnetic nanoparticle; MRgFUS = MR-guided FUS; MT = magnetic targeting; SPION = superparamagnetic iron oxide nanoparticle; TMZ = temozolomide; USgFUS = ultrasound-guided FUS.

SUBMITTED October 1, 2017. ACCEPTED November 14, 2017.

INCLUDE WHEN CITING DOI: 10.3171/2017.11.FOCUS17620. 

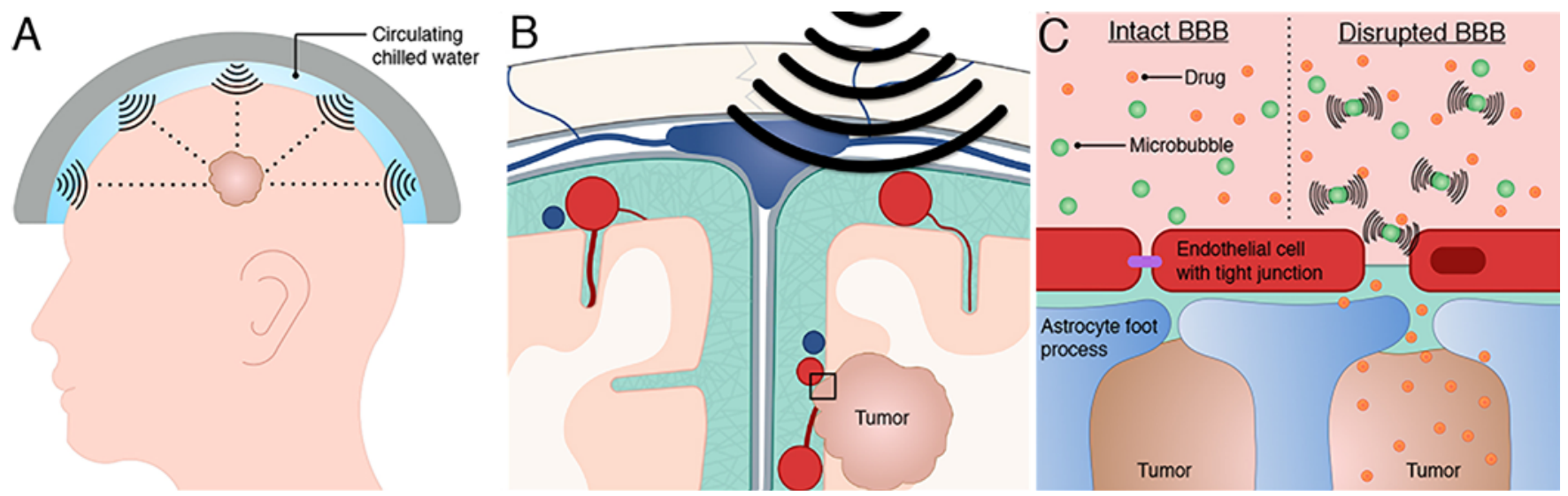

FIG. 1. A: Schematic of an ablational FUS array with beams converging at a target site. B: Schematic of an FUS beam penetrating across the calvaria toward the site of a tumor. Arteries and veins are shown as red and blue circles, respectively The boxed portion is further highlighted in panel C. C: Schematic of an intact BBB composed of endothelial cells, tight junctions, and astrocytic foot processes. An FUS beam disrupts the BBB with oscillating microbubbles, allowing penetration by therapeutics near the site of a tumor. Copyright lan D. Connolly. Published with permission.

therapeutic threshold concentration but must remain at a low concentration peripherally and within normal brain to limit side effects. Although exceptions exist, drugs that cross the BBB are typically lipid soluble, uncharged, less than $500 \mathrm{D}$, and not rapidly transported out of the CNS.45 For example, erlotinib (Tarceva) is an efficacious drug in the treatment of non-small cell lung cancer brain metastases, with all of the aforementioned properties. ${ }^{22}$ Designing effective drugs with all of these characteristics has been challenging, and recent efforts have focused on increasing CNS bioavailability through other means.

Prior attempts to enhance drug delivery have affected the brain globally and carry the potential for significant toxicity. ${ }^{13}$ Drug delivery systems, such as nanoparticles modified with brain- or tumor-targeting receptors have been used to penetrate the BBB ${ }^{23}$ Radiation therapy, commonly used for the treatment of brain tumors, disrupts the BBB by causing endothelial apoptosis. ${ }^{27}$ Intranasal injections are capable of bypassing the BBB but can only be used with subtherapeutic volumes for most drugs due to the risk of olfactory damage. ${ }^{33}$ Direct surgical injection of drugs into the brain parenchyma and spinal fluid requires large doses and carries risks of infection, toxicity, iatrogenic damage, and drug spread outside of the intended target. $^{35,36}$ Bis-chloroetheylnitrosourea (BCNU) wafers placed in the brain tumor resection cavity increase local drug delivery; however, this treatment method has associated complications and generates MRI artifact. ${ }^{6}$ Convection-enhanced delivery slowly infuses drugs using surgically placed small-diameter catheters to saturate the target site and limit regional spread. ${ }^{14}$ The limitations of these drug-to-brain delivery approaches have led to the search for safe, less invasive, and more effective alternatives. MRgFUS holds great promise for focused, repeatable disruption of the BBB to enhance systemic treatment efficacy. ${ }^{15}$

\section{MRgFUS and Microbubbles Focally Disrupt the BBB}

MRgFUS with microbubbles has been shown to transiently disrupt the BBB for up to 24 hours. ${ }^{26,38,43}$ Focused ultrasound (FUS) can cause expansion of the gaseous microbubbles until they rupture in a process termed inertial cavitation. ${ }^{43}$ Lower FUS power levels instead lead to oscillation of the microbubbles, known as stable cavitation. Stable cavitation temporarily increases the permeability of the BBB, either through mechanical disruption or by inducing physiological changes in BBB cells (Fig. 1). There is evidence for both of these mechanisms; transcellular and paracellar passage of molecules has been found with FUS. ${ }^{7}$ MRgFUS focal disruption of the BBB can increase the therapeutic concentration within a brain tumor of systemically administered drug while limiting toxicity to the normal brain and systemic organs. ${ }^{53}$ For example, MRgFUS delivered intravenous methotrexate to the brains of healthy rabbits at concentrations 13 times higher than intravenous methotrexate delivery alone $(\mathrm{p}<$ 0.01 ) and more than 3 times higher than methotrexate delivery via the internal carotid artery without MRgFUS ( $p$ $<0.01) .^{40}$ Thus, MRgFUS can reduce side effects of chemotherapeutic agents by requiring a lower systemic dose to reach a desired brain tumor tissue concentration. MRI guidance allows for not only identification of the lesion to be targeted but also dynamic feedback on the extent of BBB disruption via contrast leakage?

\section{MRgFUS and Brain Tumors MRgFUS and Microbubbles Allow Systemic Chemotherapeutics to Cross the BTB}

Several studies have suggested that the ability of MRgFUS and microbubbles to disrupt the BBB for passage of chemotherapeutics also extends to the BTB. ${ }^{29,47,59}$ In a rat brain glioma model of intravenous doxorubicin (DOX), Park et al. reported that MRgFUS increased DOX concentrations in gliomas by more than 2.5 times at 1 hour posttreatment compared with gliomas not receiving MRgFUS $(\mathrm{p}<0.01){ }^{47}$ At 24 hours posttreatment, DOX concentrations in gliomas with MRgFUS were almost 14 times higher than those without MRgFUS ( $p<0.001)$. The authors also found that gliomas with MRgFUS had no sig- 

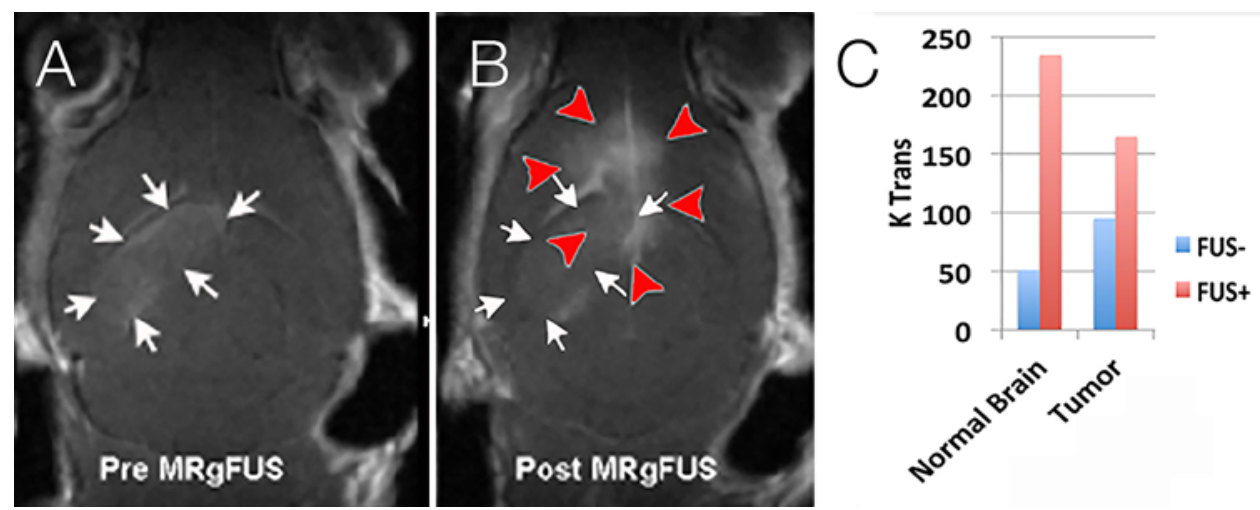

FIG. 2. A and B: Postgadolinium T1-weighted MR images obtained in a mouse, showing a brain tumor graft in the right mouse brain hemisphere (arrows) before MRgFUS application (A) and increased enhancement (arrowheads) due to BBB opening after MRgFUS (B), overlapping both brain tumor and adjacent normal tissue. C: Before MRgFUS application (FUS-), the volume transfer constant ( $\left.K^{\text {trans }}\right)$ measured from dynamic contrast-enhanced imaging was higher in the tumor than that in the normal brain tissue. After application (FUS+), BBB opening increased in both normal and tumor tissue.

nificant change in DOX concentration between 1 hour and 24 hours posttreatment. In contrast, DOX concentrations at 24 hours posttreatment in gliomas without MRgFUS were approximately $20 \%$ of the 1 -hour posttreatment levels $(p<0.01)$. This suggests that MRgFUS may not only increase the delivery of drugs across the BTB but also prolong the time on target. The only side effects reported were regions of petechiae smaller than $1 \mathrm{~mm}$ that represented extravasation of red blood cells due to blood vessel damage. The BBB and BTB may respond differently to the same dose equivalent of MRgFUS depending on the systemic treatment or tumor. In the study by Park et al. ${ }^{47}$ DOX was able to cross the BTB at higher rates than the healthy BBB, but in another study using a similar model with $\mathrm{BCNU}$, the BTB was more challenging to disrupt with MRgFUS than the BBB.${ }^{29}$ MRgFUS at the same dose equivalent increased delivery of BCNU into normal brain tissue by $340 \%$ in normal rats, but by only $202 \%$ in the tumor-implanted rats $(\mathrm{p}<0.05)$. This suggests that MRgFUS disruption of the BTB may be treatment dependent, and not directly corollary to disruption of the BBB. Figure 2 shows results from an unpublished experiment in tumorimplanted mice using MRgFUS and microbubbles, which is representative of MRgFUS in animal models.

\section{Combining Systemic Chemotherapy With MRgFUS Disruption of the BTB Improves Outcomes}

As MRgFUS disrupts the BTB and increases systemic drug penetrance, tumors are more effectively treated and survival is improved. For example, in a rat glioma model with systemic delivery of BCNU, the addition of MRgFUS to disrupt the BTB improved the median survival time compared with no treatment (53 days vs 29 days, $\mathrm{p}<$ 0.0015 ) or BCNU alone (53 days vs 32 days, significance not reported)..$^{29}$ The improvement in outcomes required concomitant delivery of systemic chemotherapy, as MRgFUS alone showed no benefit. Similar results were found with the use of temozolomide (TMZ) with MRgFUS in rat glioma, with a significantly increased median survival time over no treatment ( 23 days vs 20 days, $\mathrm{p}<0.001$ ). TMZ alone did not show a significant difference compared with no treatment ( 21 days vs 20 days, $\mathrm{p}=0.09) .{ }^{59}$
MRgFUS+TMZ slowed tumor growth significantly more than TMZ alone $(\mathrm{p}=0.002)$, with the 1 -week tumor growth being 21 times the starting volume without MRgFUS and 5 times with MRgFUS. MRgFUS improves the delivery of systemic chemotherapy and increases treatment efficacy, although the dose-response relationship has not been explored in depth.

\section{Liposome Encapsulation and MRgFUS-Enhanced Drug Delivery}

Liposome encapsulation of systemic chemotherapies effectively reduces systemic toxicity. ${ }^{34}$ This is due in part to lower levels of chemotherapy at nontumor sites, although the exact mechanism is unclear. Recent studies have combined liposome-encapsulated DOX (LeDOX) with MRgFUS-enhanced drug delivery. An early study in healthy rats showed that LeDOX paired with MRgFUS could achieve target therapeutic levels of DOX in the brain, with nontargeted brain regions having significantly lower DOX concentrations. ${ }^{55} \mathrm{~A}$ study in a rat glioma model with a single LeDOX+MRgFUS treatment showed a significantly increased median survival time compared with no treatment (31 days vs 25 days, $p<0.001$ ), whereas LeDOX alone could not produce significant results (29 days vs 25 days, $\mathrm{p}=0.17) .{ }^{56}$ Another rat glioma model used 3 LeDOX+MRgFUS treatments and reported similar median survival results for LeDOX+MRgFUS (35 days vs 18 days [no treatment], $\mathrm{p}<0.001$ ) and LeDOX alone (20 days vs 18 days [no treatment], $\mathrm{p}=0.16)$ compared with no treatment. ${ }^{3}$ Side effects were noted in the LeDOX+MRgFUS rats, including impaired activity (2 of 8 ), brain tissue damage and/or necrosis (4 of 8), and intratumoral hemorrhage ( 1 of 8 ) after 3 treatments of 5.67 $\mathrm{mg} / \mathrm{kg}$ LeDOX+MRgFUS. The only side effect noted in LeDOX-alone group was impaired activity (1 of 6). Neither study reported the significance of LeDOX+MRgFUS versus LeDOX alone. Recent evaluation of a stabilized long-circulating liposomal paclitaxel (LePTX) in a mouse model of glioblastoma showed results similar to the LeDOX studies, with a significantly improved median survival time (47 days vs 39 days, $\mathrm{p}<0.001$ ) compared with no treatment. ${ }^{51}$ Again, results were not directly compared 
with LePTX alone, but LePTX alone failed to demonstrate significance compared with no treatment (41 days vs 39 days, $\mathrm{p}=0.07$ ).

Modified liposomes have also been shown to improve MRgFUS-facilitated delivery of systemic chemotherapeutics. For example, in a mouse glioma model, LeDOX conjugated with a peptide ligand for interleukin (IL)-4 receptor, highly expressed in human brain tumors, showed a significant improvement in median survival time compared with no treatment ( 15 days vs 9 days, $p=0.0001)$ or ligand-conjugated LeDOX alone (15 days vs 3 days, $p$ $=0.017) .{ }^{60} \mathrm{DOX}$ encapsulated in cationic liposomes (cLeDOX) accumulate preferentially in tumor tissue due to their positive charge. MRgFUS+cLeDOX modestly improved median survival over cLeDOX alone in a rat glioma model (81 days vs 35 days, $p<0.001$ ), both of which were significantly greater than no treatment $(17 \pm 2$ days, $\mathrm{p}<0.001) .{ }^{28}$ Neither study compared the modified liposomes to unmodified liposomes, so it is unclear if these present an advantage.

\section{Nanoparticle Conjugation and MRgFUS}

Nanoparticles present an alternative to liposomal encapsulation, which has been shown to have its own potential side effects. ${ }^{24}$ Magnetic nanoparticle (MNP) drug delivery promises to be highly synergistic with MRgFUS. MNPs can be conjugated to chemotherapy and enriched in a targeted area by using a magnetic field generated by an external magnet to attract them, which is termed magnetic targeting (MT) ${ }^{11}$ MNPs also act as a contrast agent, potentially eliminating the need for gadolinium in MRgFUS treatment delivery. ${ }^{57}$ MNPs have difficulty crossing the BBB, but combined with MRgFUS, they can accumulate at high levels in brain tissue. ${ }^{30}$ The system operates in an open-then-pull manner, in which the BBB is first permeabilized by MRgFUS and then circulating MNPs are pulled through the permeabilized area of disrupted BBB via a magnetic field.

Preliminary studies in animals have shown promise for the combination of MNPs and MRgFUS to improve treatment delivery to brain tumors. For example, a rat model of glioma treated with epirubicin-conjugated MNP + MRgFUS+MT showed a 2.4-fold increase in MNP accumulation compared with the contralateral hemisphere, whereas MNP+MRgFUS without MT achieved a 1.2fold increase (significance not reported). ${ }^{30}$ This increase in MNP accumulation led to a 16-fold increase in the concentration of epirubicin in MRgFUS+MT-treated brain tissue, compared with MRgFUS alone (significance not reported). The MNP+MRgFUS+MT-treated group showed prolonged survival time compared with no treatment (31 days vs 18 days, $\mathrm{p}=0.0002$ ) or $\mathrm{MNP}+\mathrm{MRgFUS}$ without MT (30.5 days vs 20 days, significance not reported). Tumor growth over 7 days was also delayed in the MNP+MRgFUS+MT group compared with no treatment $(106 \% \pm 24 \%$ vs $313 \% \pm 103 \%$, significance not reported). The authors noted, however, that performing MT within the longer distances of human anatomy would likely require a superconducting magnetic coil or MNPs that are more strongly magnetic. Similar conclusions were found when using BCNU. ${ }^{10}$ Both MRgFUS alone and MT alone increased MNP brain concentration by 2-fold in the treated compared with the untreated brain regions, but $\mathrm{MNP}+\mathrm{MRgFUS}+\mathrm{MT}$ increased MNP accumulation by almost 10-fold compared with the untreated region and 26-fold compared with MNP without additional treatment (significance not reported). The mean change in tumor volume at 1 week posttreatment showed that the mediumdose MNP+MRgFUS+MT group effectively experienced delayed tumor progression compared with no treatment $\left(-0.79 \pm 0.35 \mathrm{~cm}^{3}\right.$ vs $\left.2.98 \pm 2.61 \mathrm{~cm}^{3}, \mathrm{p}<0.05\right)$ or MNP alone $\left(-0.79 \pm 0.35 \mathrm{~cm}^{3}\right.$ vs $2.96 \pm 3.00 \mathrm{~cm}^{3}$, significance not reported). In addition, MNP alone was superior to unbound BCNU $\left(1.15 \pm 1.58 \mathrm{~cm}^{3}\right.$ vs $2.48 \pm 3.09 \mathrm{~cm}^{3}$, significance not reported). Results from low- and high-dose MNPs demonstrated that this effect was dose dependent.

Efforts at further simplifying MNP-containing protocols have resulted in microbubbles that are conjugated with superparamagnetic iron oxide nanoparticles (SPIONs, a type of MNP) and loaded with DOX. ${ }^{19}$ Thus, a single substance combines the functionality of microbubbles, gadolinium contrast, and chemotherapy-conjugated MNPs with delivery enhanced by MRgFUS+MT. In a rat glioma model, SPION deposition in MT+MRgFUS increased by 4-fold ( $\mathrm{p}<0.01)$ compared with the contralateral, nontargeted hemisphere. MRgFUS alone and MT only enhanced accumulation by 2.7 -fold $(\mathrm{p}<0.01)$ and 2.3 -fold $(\mathrm{p}<0.05)$, respectively. Similarly, the MT+MRgFUS group increased DOX deposition in the treated hemisphere by 2 -fold $(\mathrm{p}<$ $0.05)$ compared with the control hemisphere. The followup study used a different formulation that improved DOXcarrying capacity and R2 relaxivity, thus allowing the authors to track tissue accumulation more accurately via MR relaxometry. ${ }^{17}$ SPION-DOX complexes accumulated in MRgFUS-targeted brain tissue at a 2.8 -fold $(\mathrm{p}<0.05)$ higher concentration when MT was applied versus without it. Likewise, DOX deposition into targeted brain tissue was enhanced by more than 2.1-fold $(\mathrm{p}<0.05)$ with MT. The authors found that the MR R2 value and SPION concentration were highly correlated $\left(\mathrm{R}^{2}=0.83\right)$, and furthermore, SPION concentration was highly correlated $\left(\mathrm{R}^{2}=\right.$ 0.79 ) with DOX accumulation. The combination SPIONDOX microbubbles may prove useful due to the ease with which dosing could be monitored through imaging.

\section{Chemotherapy With Modified Microbubbles and Ultrasound-Guided FUS}

Microbubble-encapsulated BCNU used with ultrasound-guided FUS (USgFUS) prolonged the half-life and reduced peripheral uptake of $\mathrm{BCNU}$, decreasing tumor progression in glioma rats. ${ }^{54}$ In particular, liver deposition of BCNU 10 minutes after treatment with microbubble-encapsulated BCNU was 9-fold than that of BCNU only (significance not reported), whereas encapsulated BCNU with FUS was 5-fold less than that of BCNU (p $<0.01$, with FUS). Another study showed that USgFUS could oscillate BCNU-loaded microbubbles at their resonant frequency, producing stable cavitation and reducing the chance of red blood cell extravasation..$^{18}$ The median survival was improved compared with no treatment (28 days vs 18 days, $\mathrm{p}=0.001$ ), but no comparison was made with free BCNU with unmodified microbubbles. Micro- 
bubbles can be further modified with moieties designed to target tumor markers like VEGF, which one study reported could be used with USgFUS to prolong survival in rat glioma. ${ }^{20}$ The experimental group had increased median survival compared with no treatment (42 days vs 19 days, $\mathrm{p}=0.007$ ) or BCNU alone (42 days vs 23 days, significance not reported). Notably, the nontargeted BCNUloaded microbubbles with FUS did not have a significant increase in median survival compared with no treatment. Liver accumulation was 3-fold less in the VEGF-targeted experimental group than in the nontargeted group ( $\mathrm{p}<$ $0.05)$. Whether chemotherapy is loaded onto liposomes, MNPs, or microbubbles, tumor-targeting moieties will be a part of future MRgFUS-coupled drug delivery systems.

\section{Immunotherapy and MRgFUS}

Research on immunotherapy usage with MRgFUS for brain tumors has largely been limited to delivery of HER2-targeting antibodies for treatment of breast cancer metastases. In a mouse model, FUS treatment effectively delivered trastuzumab, with dose limited by red blood cell extravasation. ${ }^{25}$ Without MRgFUS, brain tissue levels of trastuzumab were undetectable in all but one case. In a rat model of HER2-positive human breast cancer metastases to the brain, Park et al. reported that MRgFUSdelivered trastuzumab increased the median survival time compared with no treatment (83 days vs 63 days, $\mathrm{p}$ $=0.008)$ and trastuzumab only (83 days vs 71 days, not significant), with a significant reduction in tumor volume $(p<0.05$ at week 7$)$ compared with all other groups. ${ }^{46}$ Of note, this effect was derived from a subset of the experimental group, as 6 of 10 experimental mice were nonresponders. Rats treated with MRgFUS and peripherally administered HER2-specific NK-92 cells showed a longer mean survival time (times unreported, $\mathrm{p}<0.05$ ). ${ }^{2}$ Similar to the study by Park et al., ${ }^{46}$ about half of the treatment animals were nonresponders, following a survival curve similar to animals without treatment. The study found no histological signs of red blood cell extravasation, although the authors noted that a week or more elapsed between the last MRgFUS treatment and euthanization. In a rat glioma model, FUS increased the intraperitoneal IL-12 concentration in the brain by almost 2.9 -fold $(\mathrm{p}=0.003)$ versus without FUS. ${ }^{9}$ The IL-12 + FUS significantly increased T-lymphocyte presence inside the tumors compared with sham treatment $\left(\mathrm{CD}^{+}{ }^{+} \mathrm{CD} 4^{+}:>4\right.$-fold, $\mathrm{p}<$ 0.001; $\mathrm{CD}^{+} \mathrm{CD}^{+}$: 5 -fold, $\mathrm{p}<0.01 ; \mathrm{CD}^{+}{ }^{+} \mathrm{CD} 25^{+}:$2-fold, $\mathrm{p}$ $<0.05$; cytotoxic-to-regulatory T-cell ratio increase: 2.5 fold, $p<0.001)$. This effect was not seen in healthy rats or systemically in tumor-implanted rats. Median survival time was increased compared with no treatment (30 days vs 21 days, $\mathrm{p}<0.001$ ) and IL-12 alone (30 days vs 26 days, significance not reported). Immunotherapies could be rapidly integrated into MRgFUS protocols, should targeting in animal models continue to show promise.

\section{Gene Therapy and USgFUS}

FUS as a means to deliver DNA-loaded microbubbles is an emerging field. Folate-conjugated cationic microbubbles (cMBs) containing DNA combined with ultrasound targeting successfully transfected tumor cells in a rat glioma model. ${ }^{16}$ Treatment with FUS and folate-conjugated cMBs improved reporter gene expression in tumor tissue by 4.7fold compared with direct injection $(\mathrm{p}<0.01)$ and 1.5 -fold (significance not reported) compared with cMBs without folate conjugation. Expression was only found within the tumor. An additional study in a rat glioma model used DNA-loaded cMBs conjugated with VEGFR2-targeted monoclonal antibodies. ${ }^{8}$ Reporter gene expression in tissue receiving targeted or nontargeted cMBs + FUS was 3.7fold $(\mathrm{p}<0.01)$ and 2.3 -fold $(\mathrm{p}<0.05)$ higher, respectively, than direct DNA injection into the tumor. The authors then tested the cMBs with a suicide gene previously tested with ultrasound, $p H S V-T K$, which converts ganciclovir into a product that terminates DNA replication and results in tumor death. ${ }^{61}$ The cMBs+FUS decreased tumor volume at day 25 compared with direct injection $\left(9.7 \pm 5.2 \mathrm{~mm}^{3}\right.$ vs $40.1 \pm 4.3 \mathrm{~mm}^{3}$, significance not reported) and nontargeted cMBs $\left(9.7 \pm 5.2 \mathrm{~mm}^{3}\right.$ vs $21.8 \pm 4.7 \mathrm{~mm}^{3}$, significance not reported). Both targeted and nontargeted cMBs had significantly decreased tumor volume $(\mathrm{p}<0.01)$ and increased median survival time $(\mathrm{p}<0.05)$ compared with untreated controls. Among brain tumor therapeutics used with FUS, gene therapy is the least developed. Incorporation of MR guidance as this technology nears clinical trials will be necessary for imaging through the human skull.

\section{Limitations}

Promising preclinical studies in animal models will need to be tested thoroughly in humans. A Phase 1 clinical trial should be conducted to evaluate toxicity and determine the safe levels for MRgFUS strength, and the choice, dosing, and timing of systemic chemotherapeutic agents. Compared with rodents, the thicker human skull and the longer distances to any given point within the human cranium will attenuate ultrasound, requiring higher power. Likewise, tumor depth may limit which types of lesions could be reasonably treated. ${ }^{39}$ Severe or symptomatic peritumoral edema may be exacerbated by further disruption of the BTB. Setups with stereotactic frames would be difficult to use in patients requiring multiple FUS treatments, but frameless designs, such as the NaviFUS system, are being developed. ${ }^{31} \mathrm{~A}$ risk of hemorrhage has been documented in animal models, but it is uncertain how this will translate to humans. ${ }^{25,47}$ The only relevant study in humans is a nonrandomized, single-arm Phase 1 clinical trial (registration no. NCT02986932, clinicaltrials.gov) evaluating the safety of BBB opening with FUS in 6 patients with Alzheimer's disease. Patients will be followed out to 2 months after opening a $3 \times 3-\mathrm{cm}$ area of BBB. Results have not yet been released. Experiences with high-intensity focused ultrasound in the ablation of brain tumors in human patients have yielded minimal side effects other than pain symptoms possibly related to dural heating. ${ }^{12,39}$

\section{Conclusions}

MRgFUS holds significant promise for less invasive, repeatable, targeted drug delivery to brain tumors. It may act synergistically with drug delivery technologies that can further enhance delivery and treatment release within the desired tissue, including nanoparticles and liposomes. In 
animal models, MRgFUS has been shown to successfully enhance the delivery of chemotherapy, immunotherapy, and gene therapy with corresponding improvements in treatment efficiency, tumor progression, and overall survival.

\section{References}

1. Abbott NJ, Rönnbäck L, Hansson E: Astrocyte-endothelial interactions at the blood-brain barrier. Nat Rev Neurosci 7:41-53, 2006

2. Alkins R, Burgess A, Kerbel R, Wels WS, Hynynen K: Early treatment of HER2-amplified brain tumors with targeted NK92 cells and focused ultrasound improves survival. Neuro Oncol 18:974-981, 2016

3. Aryal M, Vykhodtseva N, Zhang YZ, Park J, McDannold N: Multiple treatments with liposomal doxorubicin and ultrasound-induced disruption of blood-tumor and blood-brain barriers improve outcomes in a rat glioma model. J Control Release 169:103-111, 2013

4. Ballabh P, Braun A, Nedergaard M: The blood-brain barrier: an overview: structure, regulation, and clinical implications. Neurobiol Dis 16:1-13, 2004

5. Bauer R, Martin E, Haegele-Link S, Kaegi G, von Specht M, Werner B: Noninvasive functional neurosurgery using transcranial MR imaging-guided focused ultrasound. Parkinsonism Relat Disord 20 (Suppl 1):S197-S199, 2014

6. Bregy A, Shah AH, Diaz MV, Pierce HE, Ames PL, Diaz $\mathrm{D}$, et al: The role of Gliadel wafers in the treatment of highgrade gliomas. Expert Rev Anticancer Ther 13:1453-1461, 2013

7. Burgess A, Hynynen K: Drug delivery across the blood-brain barrier using focused ultrasound. Expert Opin Drug Deliv 11:711-721, 2014

8. Chang EL, Ting CY, Hsu PH, Lin YC, Liao EC, Huang CY, et al: Angiogenesis-targeting microbubbles combined with ultrasound-mediated gene therapy in brain tumors. J Control Release 255:164-175, 2017

9. Chen PY, Hsieh HY, Huang CY, Lin CY, Wei KC, Liu HL: Focused ultrasound-induced blood-brain barrier opening to enhance interleukin-12 delivery for brain tumor immunotherapy: a preclinical feasibility study. J Transl Med 13:93, 2015

10. Chen PY, Liu HL, Hua MY, Yang HW, Huang CY, Chu PC, et al: Novel magnetic/ultrasound focusing system enhances nanoparticle drug delivery for glioma treatment. Neuro Oncol 12:1050-1060, 2010

11. Chertok B, Moffat BA, David AE, Yu F, Bergemann C, Ross $\mathrm{BD}$, et al: Iron oxide nanoparticles as a drug delivery vehicle for MRI monitored magnetic targeting of brain tumors. Biomaterials 29:487-496, 2008

12. Coluccia D, Fandino J, Schwyzer L, O’Gorman R, Remonda L, Anon J, et al: First noninvasive thermal ablation of a brain tumor with MR-guided focused ultrasound. J Ther Ultrasound 2:17, 2014

13. De Bock M, Wang N, Decrock E, Bol M, Gadicherla AK, Culot M, et al: Endothelial calcium dynamics, connexin channels and blood-brain barrier function. Prog Neurobiol 108:1-20, 2013

14. Debinski W, Tatter SB: Convection-enhanced delivery for the treatment of brain tumors. Expert Rev Neurother 9:15191527,2009

15. Downs ME, Buch A, Sierra C, Karakatsani ME, Teichert $\mathrm{T}$, Chen S, et al: Long-term safety of repeated blood-brain barrier opening via focused ultrasound with microbubbles in non-human primates performing a cognitive task. PLoS One 10:e0125911, 2015 (Erratum in PLoS One 10:e0130860, 2015)

16. Fan $\mathrm{CH}$, Chang EL, Ting CY, Lin YC, Liao EC, Huang CY, et al: Folate-conjugated gene-carrying microbubbles with focused ultrasound for concurrent blood-brain barrier opening and local gene delivery. Biomaterials 106:46-57, 2016

17. Fan CH, Cheng YH, Ting CY, Ho YJ, Hsu PH, Liu HL, et al: Ultrasound/magnetic targeting with SPIO-DOX-microbubble complex for image-guided drug delivery in brain tumors. Theranostics 6:1542-1556, 2016

18. Fan CH, Ting CY, Chang YC, Wei KC, Liu HL, Yeh CK: Drug-loaded bubbles with matched focused ultrasound excitation for concurrent blood-brain barrier opening and braintumor drug delivery. Acta Biomater 15:89-101, 2015

19. Fan CH, Ting CY, Lin HJ, Wang CH, Liu HL, Yen TC, et al: SPIO-conjugated, doxorubicin-loaded microbubbles for concurrent MRI and focused-ultrasound enhanced brain-tumor drug delivery. Biomaterials 34:3706-3715, 2013

20. Fan CH, Ting CY, Liu HL, Huang CY, Hsieh HY, Yen TC, et al: Antiangiogenic-targeting drug-loaded microbubbles combined with focused ultrasound for glioma treatment. Biomaterials 34:2142-2155, 2013

21. Fasano A, Llinas M, Munhoz RP, Hlasny E, Kucharczyk W, Lozano AM: MRI-guided focused ultrasound thalamotomy in non-ET tremor syndromes. Neurology 89:771-775, 2017

22. Fukudo M, Ikemi Y, Togashi Y, Masago K, Kim YH, Mio $\mathrm{T}$, et al: Population pharmacokinetics/pharmacodynamics of erlotinib and pharmacogenomic analysis of plasma and cerebrospinal fluid drug concentrations in Japanese patients with non-small cell lung cancer. Clin Pharmacokinet 52:593609, 2013

23. Gabathuler R: Approaches to transport therapeutic drugs across the blood-brain barrier to treat brain diseases. Neurobiol Dis 37:48-57, 2010

24. Iwamoto T: Clinical application of drug delivery systems in cancer chemotherapy: review of the efficacy and side effects of approved drugs. Biol Pharm Bull 36:715-718, 2013

25. Kinoshita M, McDannold N, Jolesz FA, Hynynen K: Noninvasive localized delivery of Herceptin to the mouse brain by MRI-guided focused ultrasound-induced blood-brain barrier disruption. Proc Natl Acad Sci U S A 103:11719-11723, 2006

26. Kovacs ZI, Kim S, Jikaria N, Qureshi F, Milo B, Lewis BK, et al: Disrupting the blood-brain barrier by focused ultrasound induces sterile inflammation. Proc Natl Acad Sci U S A 114:E75-E84, 2017

27. Li YQ, Chen P, Haimovitz-Friedman A, Reilly RM, Wong CS: Endothelial apoptosis initiates acute blood-brain barrier disruption after ionizing radiation. Cancer Res 63:59505956, 2003

28. Lin Q, Mao KL, Tian FR, Yang JJ, Chen PP, Xu J, et al: Brain tumor-targeted delivery and therapy by focused ultrasound introduced doxorubicin-loaded cationic liposomes. Cancer Chemother Pharmacol 77:269-280, 2016

29. Liu HL, Hua MY, Chen PY, Chu PC, Pan CH, Yang HW, et al: Blood-brain barrier disruption with focused ultrasound enhances delivery of chemotherapeutic drugs for glioblastoma treatment. Radiology 255:415-425, 2010

30. Liu HL, Hua MY, Yang HW, Huang CY, Chu PC, Wu JS, et al: Magnetic resonance monitoring of focused ultrasound/ magnetic nanoparticle targeting delivery of therapeutic agents to the brain. Proc Natl Acad Sci U S A 107:1520515210,2010

31. Liu HL, Jan CK, Chu PC, Hong JC, Lee PY, Hsu JD, et al: Design and experimental evaluation of a 256-channel dualfrequency ultrasound phased-array system for transcranial blood-brain barrier opening and brain drug delivery. IEEE Trans Biomed Eng 61:1350-1360, 2014

32. Liu Y, Lu W: Recent advances in brain tumor-targeted nanodrug delivery systems. Expert Opin Drug Deliv 9:671-686, 2012

33. Lochhead JJ, Thorne RG: Intranasal delivery of biologics to 
the central nervous system. Adv Drug Deliv Rev 64:614 628,2012

34. Malam Y, Loizidou M, Seifalian AM: Liposomes and nanoparticles: nanosized vehicles for drug delivery in cancer. Trends Pharmacol Sci 30:592-599, 2009

35. Marks WJ Jr, Bartus RT, Siffert J, Davis CS, Lozano A, Boulis N, et al: Gene delivery of AAV2-neurturin for Parkinson's disease: a double-blind, randomised, controlled trial. Lancet Neurol 9:1164-1172, 2010

36. Marks WJ Jr, Ostrem JL, Verhagen L, Starr PA, Larson PS, Bakay RA, et al: Safety and tolerability of intraputaminal delivery of CERE-120 (adeno-associated virus serotype 2-neurturin) to patients with idiopathic Parkinson's disease: an open-label, phase I trial. Lancet Neurol 7:400-408, 2008

37. Mathieu D, Kondziolka D, Cooper PB, Flickinger JC, Niranjan A, Agarwala S, et al: Gamma knife radiosurgery in the management of malignant melanoma brain metastases. Neurosurgery 60:471-482, 2007

38. McDannold N, Arvanitis CD, Vykhodtseva N, Livingstone MS: Temporary disruption of the blood-brain barrier by use of ultrasound and microbubbles: safety and efficacy evaluation in rhesus macaques. Cancer Res 72:3652-3663, 2012

39. McDannold N, Clement GT, Black P, Jolesz F, Hynynen K: Transcranial magnetic resonance imaging- guided focused ultrasound surgery of brain tumors: initial findings in 3 patients. Neurosurgery 66:323-332, 2010

40. Mei J, Cheng Y, Song Y, Yang Y, Wang F, Liu Y, et al: Experimental study on targeted methotrexate delivery to the rabbit brain via magnetic resonance imaging-guided focused ultrasound. J Ultrasound Med 28:871-880, 2009

41. Miller DB, O'Callaghan JP: New horizons for focused ultrasound (FUS) - therapeutic applications in neurodegenerative diseases. Metabolism 69 Suppl:S3-S7, 2017

42. Mizee MR, de Vries HE: Blood-brain barrier regulation: Environmental cues controlling the onset of barrier properties. Tissue Barriers 1:e26882, 2013

43. Okada K, Kudo N, Niwa K, Yamamoto K: A basic study on sonoporation with microbubbles exposed to pulsed ultrasound. J Med Ultrason 32:3-11, 2005

44. Ostrom QT, Gittleman H, Xu J, Kromer C, Wolinsky Y, Kruchko C, et al: CBTRUS Statistical Report: Primary brain and other central nervous system tumors diagnosed in the United States in 2009-2013. Neuro Oncol 18 (Suppl 5):v1v75, 2016

45. Pardridge WM: The blood-brain barrier: bottleneck in brain drug development. NeuroRx 2:3-14, 2005

46. Park EJ, Zhang YZ, Vykhodtseva N, McDannold N: Ultrasound-mediated blood-brain/blood-tumor barrier disruption improves outcomes with trastuzumab in a breast cancer brain metastasis model. J Control Release 163:277-284, 2012

47. Park J, Aryal M, Vykhodtseva N, Zhang YZ, McDannold N: Evaluation of permeability, doxorubicin delivery, and drug retention in a rat brain tumor model after ultrasound-induced blood-tumor barrier disruption. J Control Release 250:7785,2017

48. Prados MD, Byron SA, Tran NL, Phillips JJ, Molinaro AM, Ligon KL, et al: Toward precision medicine in glioblastoma: the promise and the challenges. Neuro Oncol 17:1051-1063, 2015

49. Puhalla S, Elmquist W, Freyer D, Kleinberg L, Adkins C, Lockman P, et al: Unsanctifying the sanctuary: challenges and opportunities with brain metastases. Neuro Oncol 17:639-651, 2015

50. Ravikumar VK, Parker JJ, Hornbeck TS, Santini VE, Pauly $\mathrm{KB}$, Wintermark M, et al: Cost-effectiveness of focused ultrasound, radiosurgery, and DBS for essential tremor. Mov Disord 32:1165-1173, 2017

51. Shen Y, Pi Z, Yan F, Yeh CK, Zeng X, Diao X, et al: Enhanced delivery of paclitaxel liposomes using focused ul- trasound with microbubbles for treating nude mice bearing intracranial glioblastoma xenografts. Int J Nanomedicine 12:5613-5629, 2017

52. Staudt M, Lasithiotakis K, Leiter U, Meier F, Eigentler T, Bamberg M, et al: Determinants of survival in patients with brain metastases from cutaneous melanoma. Br J Cancer 102:1213-1218, 2010

53. Thévenot E, Jordão JF, O'Reilly MA, Markham K, Weng YQ, Foust KD, et al: Targeted delivery of self-complementary adeno-associated virus serotype 9 to the brain, using magnetic resonance imaging-guided focused ultrasound. Hum Gene Ther 23:1144-1155, 2012

54. Ting CY, Fan CH, Liu HL, Huang CY, Hsieh HY, Yen TC, et al: Concurrent blood-brain barrier opening and local drug delivery using drug-carrying microbubbles and focused ultrasound for brain glioma treatment. Biomaterials 33:704-712, 2012

55. Treat LH, McDannold N, Vykhodtseva N, Zhang Y, Tam K, Hynynen K: Targeted delivery of doxorubicin to the rat brain at therapeutic levels using MRI-guided focused ultrasound. Int J Cancer 121:901-907, 2007

56. Treat LH, McDannold N, Zhang Y, Vykhodtseva N, Hynynen $\mathrm{K}$ : Improved anti-tumor effect of liposomal doxorubicin after targeted blood-brain barrier disruption by MRI-guided focused ultrasound in rat glioma. Ultrasound Med Biol 38:1716-1725, 2012

57. Veiseh O, Gunn JW, Zhang M: Design and fabrication of magnetic nanoparticles for targeted drug delivery and imaging. Adv Drug Deliv Rev 62:284-304, 2010

58. Watkins S, Robel S, Kimbrough IF, Robert SM, Ellis-Davies G, Sontheimer H: Disruption of astrocyte-vascular coupling and the blood-brain barrier by invading glioma cells. Nat Commun 5:4196, 2014

59. Wei KC, Chu PC, Wang HYJ, Huang CY, Chen PY, Tsai HC, et al: Focused ultrasound-induced blood-brain barrier opening to enhance temozolomide delivery for glioblastoma treatment: a preclinical study. PLoS One 8:e58995, 2013

60. Yang FY, Wong TT, Teng MC, Liu RS, Lu M, Liang HF, et al: Focused ultrasound and interleukin-4 receptor-targeted liposomal doxorubicin for enhanced targeted drug delivery and antitumor effect in glioblastoma multiforme. J Control Release 160: $652-658,2012$

61. Zhou XL, Shi YL, Li X: Inhibitory effects of the ultrasoundtargeted microbubble destruction-mediated herpes simplex virus-thymidine kinase/ganciclovir system on ovarian cancer in mice. Exp Ther Med 8:1159-1163, 2014

\section{Disclosures}

The authors report no conflict of interest concerning the materials or methods used in this study or the findings specified in this paper.

\section{Author Contributions}

Conception and design: Hayden Gephart, Wintermark. Acquisition of data: Lamsam, Johnson, Connolly. Analysis and interpretation of data: Lamsam, Johnson, Connolly. Drafting the article: Lamsam, Johnson, Connolly. Critically revising the article: Lamsam, Wintermark. Reviewed submitted version of manuscript: Hayden Gephart. Approved the final version of the manuscript on behalf of all authors: Hayden Gephart. Study supervision: Hayden Gephart, Lamsam, Wintermark.

\section{Correspondence}

Melanie Hayden Gephart: Stanford University Medical Center, Stanford, CA.mghayden@stanford.edu. 